\title{
Global and depth resolved phosphorus magnetic resonance spectroscopy to predict outcome after birth asphyxia
}

Department of
Paediatrics and
Medical Research
Council Biochemical
and Clinical Magnetic
Resonance Unit,
John Radcliffe Hospital,
Oxford
J Moorcraft
N M Bolas
N K Ives
R Ouwerkerk
J Smyth
B Rajagopalan
P L Hope
G K Radda
Correspondence to:
Dr P Hope,
Special Care Nursery,
John Radcliffe
Maternity Hospital,
Headington,
Oxford OX3 9DU.
Accepted 18 July 1991

\author{
J Moorcraft, N M Bolas, N K Ives, R Ouwerkerk, J Smyth, B Rajagopalan, P L Hope, \\ G K Radda
}

handicap in a larger group of asphyxiated infants.

\section{Patients and methods}

Full details of the MRS methods are described elsewhere. ${ }^{5}$ Studies were performed without sedation over a period of $30-45$ minutes, usually after a feed. Major movements of the infant's head position during PMRFI were unusual, but did necessitate restarting data collection. The infant was positioned prone in the bore of a 1.9 tesla superconducting magnet, with the right side of the head resting on a foam pillow into which a surface coil was incorporated. The surface coil consisted of a $15 \mathrm{~cm}$ diameter transmitter and a $6.5 \mathrm{~cm}$ diameter receiver coil, and received ${ }^{31} \mathrm{P}$ MRS signals at a frequency of $32.7 \mathrm{MHz}$ from temporoparietal brain. Two conventional 'pulse and collect' sequences (64 pulses, duration $275 \mu$ secs, interval 3s) were recorded and, after profile correction and Fourier transformation, a 'global' spectrum was obtained. Figure 1(a) shows a typical global spectrum from a normal infant, with the peak assignments, and fig 2(a) shows a comparable global spectrum from a severely asphyxiated infant. Relative metabolite concentrations were calculated from spectra using a computer assisted curve fitting programme.

Depth resolved data were then obtained using PMRFI, which utilises the linear radiofrequency field produced by the transmitter coil to spatially encode spectral data. ${ }^{6}$ Three spectra were taken for analysis from each infant, from the data matrix produced by PMRFI. Each spectrum is derived from an approximately disc shaped volume of brain. Approximately $90 \%$ of the signal contributing to each spectrum comes from a disc of $1 \mathrm{~cm}$ depth and the same diameter as the receiver coil. Superficial, middle, and deep spectra were analysed, corresponding to signal from brain approximately $0-1,1-2$, and $2-3 \mathrm{~cm}$ below the skull. Figures 1(b), (c), and (d) show depth resolved spectra from a normal newborn and figs 2(b), (c), and (d) are comparable spectra from a severely asphyxiated infant.

Complete global and depth resolved data sets were recorded from 12 normal infants, gestation 38-42 weeks, Apgar scores 5-10 at 1 minute and 9-10 at 5 minutes, studied at $1-5$ days old. Similar data were recorded from 32 infants studied after birth asphyxia. The table shows clinical details of the asphyxiated infants. Because of the small number of normal studies, values for metabolite ratios from asphyxiated infants were compared with the entire range of 

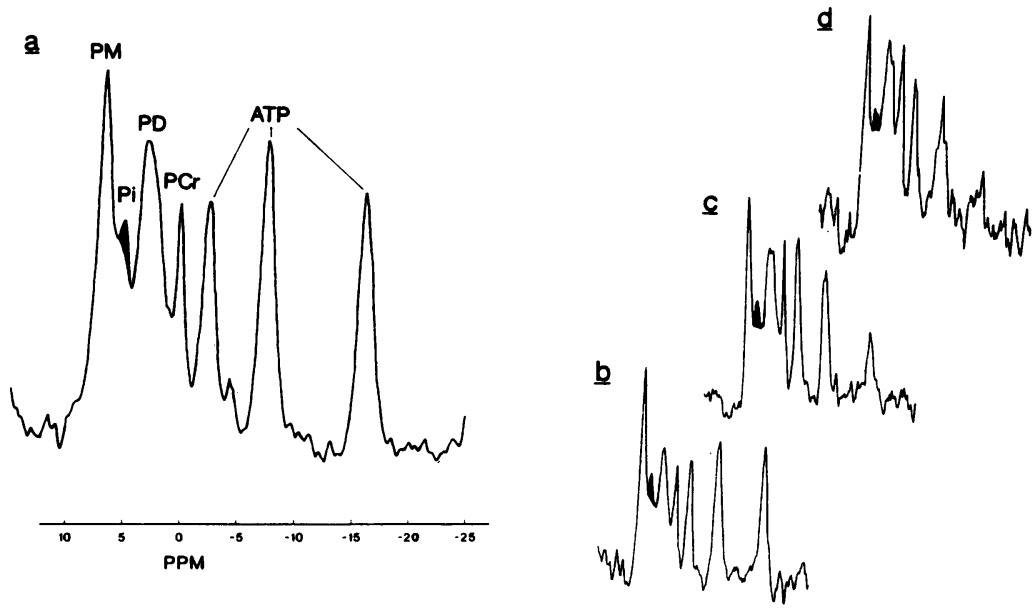

Figure 1 Global (a), and spatially localised magnetic resonance spectra from superficial $(b)$, middle (c), and deep (d) regions of the brain of a normal infant. See text for details of localisation. Peak assignments are-ATP: adenosine triphosphate, $P C r=$ phosphocreatine, $P D=$ phosphodiesters and phospholipid bilayers, $P i=$ inorganic orthophosphate, $P M=$ phosphomonoesters. The three ATP peaks are $\beta, \alpha$, and $\gamma$ from right to left. $\beta$ ATP may be underestimated on regional data for technical reasons.

a

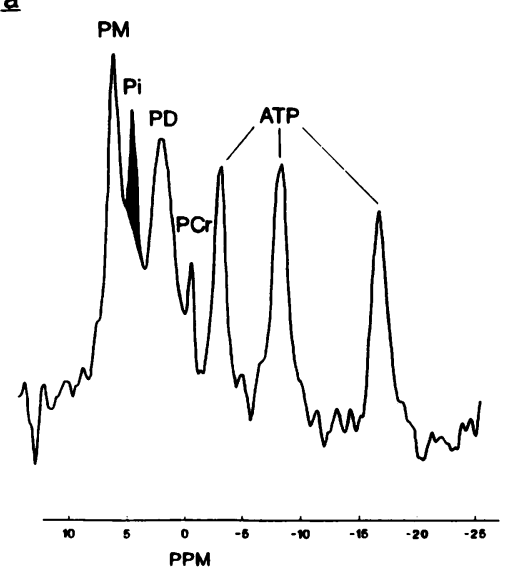

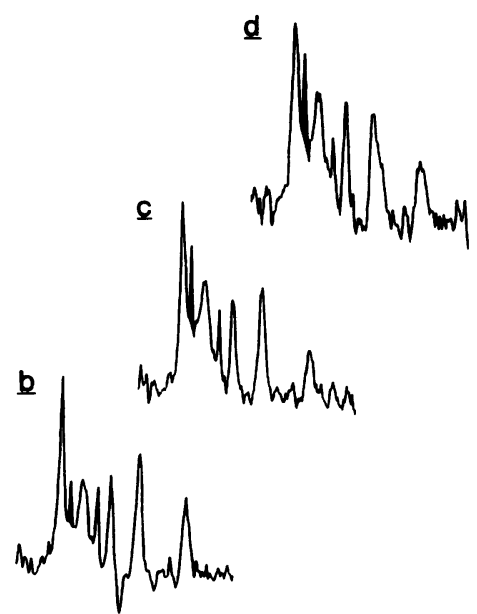

Figure 2 Global $(a)$, and spatially localised $(b),(c)$, and $(d)$ magnetic resonance data from the same regions as fig 1 , from an asphyxiated baby with an adverse outcome studied at 4 days. Peak assignments are-ATP:adenosine triphosphate, PCr: phosphocreatine, $P D$ : phosphodiesters and phospholipid bilayers, $P i=$ inorganic orthophosphate,

PM: phosphomonoesters. The three ATP peaks are $\beta, \alpha$, and $\gamma$ from right to left. $\beta$ ATP may be underestimated on regional data for technical reasons.

Clinical details of asphyxiated infants. Results are median (range)

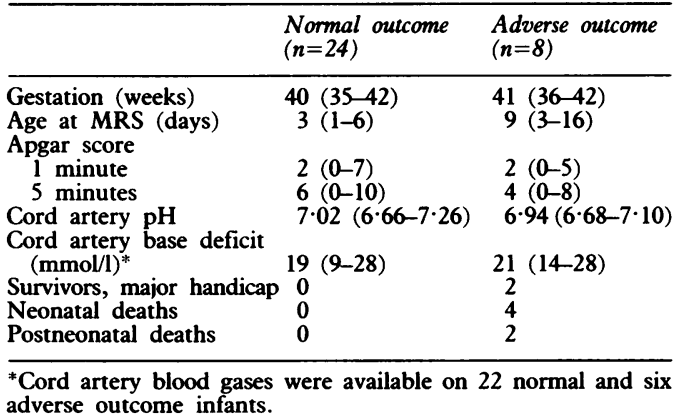

values from normals, rather than using standard deviations or confidence intervals.

All but two of the asphyxiated infants who survived the neonatal period were assessed neurodevelopmentally at regular intervals in hospital follow up clinics. The other two infants did not attend the hospital clinic but were assessed as completely normal on health visitor assessment at 18 months.
The study was approved by the Central Oxford Research Ethics Committee and the parents of all infants gave informed consent. Normal infants were recruited by discussing the project with groups of parents during antenatal classes. These parents were invited to contact the investigators via their midwife in the postnatal period.

\section{Results}

Eight asphyxiated infants had an adverse outcome. Four died in the neonatal period and two others, both severely neurologically abnormal, died at 11 weeks and 19 months. Two survived with major cognitive deficits and spastic quadriplegia at 37 and 38 months. Twenty four asphyxiated infants, now age $15-43$ (median 31 ) months were neurodevelopmentally normal at their last assessment at 9-19 (median 13) months.

Global $\mathrm{PCr} / \mathrm{Pi}$ and $\mathrm{Pi} / \mathrm{ATP}$ ratios for all infants are shown in fig 3(a) and (b), which

Global PCr/PI

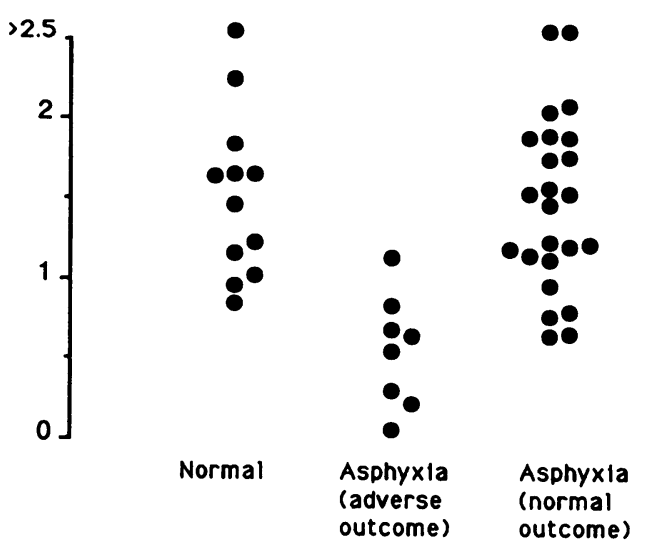

Figure 3 (a) Global PCr/Pi ratios from normal infants and asphyxiated infants divided into groups according to outcome. Infants with adverse outcome differ from normals (Mann-Whitney test, $p<0 \cdot 002$ ) and from asphyxiated infants with good outcome $(p<0.001)$.

\section{Global PI/ATP}

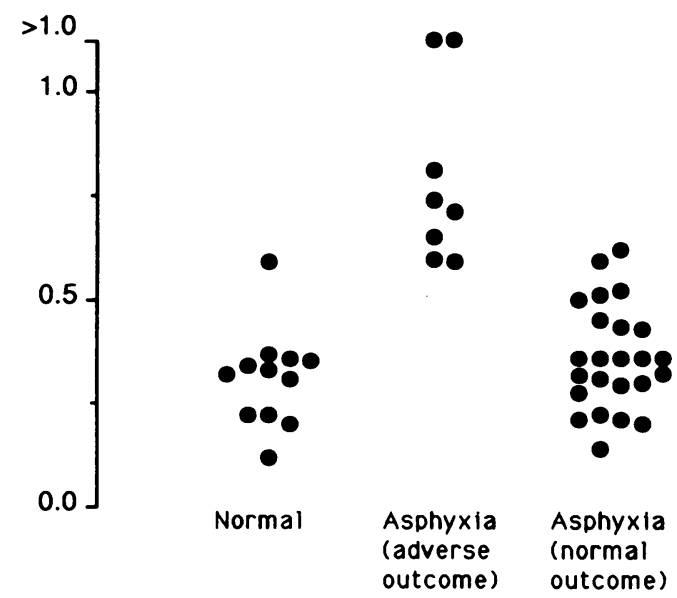

Figure 3 (b) Global Pi/ATP ratios from normal infants and asphyxiated infants divided into groups according to outcome. Infants with adverse outcome differ from normals (Mann-Whitney test, $p<0 \cdot 002$ ), and from asphyxiated infants with good outcome $(p<0 \cdot 001)$. 
show no significant differences in metabolite ratios between normal infants and those asphyxiated babies with a good outcome. The infants with adverse outcome form a population with a lower PCr/Pi and higher $\mathrm{Pi} / \mathrm{ATP}$ ratio. Only one asphyxiated infant with adverse outcome had $\mathrm{PCr} / \mathrm{Pi}$ in the range of normals. There were fewer 'false positive' results from infants with a normal outcome using $\mathrm{Pi} / \mathrm{ATP}$ ratio as a predictor. Sensitivity, specificity, and positive predictive value for the prediction of death or handicap were $88 \%, 83 \%$, and $64 \%$ respectively for $\mathrm{PCr} / \mathrm{Pi}$ and $88 \%, 96 \%$, and $88 \%$ for $\mathrm{Pi} / \mathrm{ATP}$.

Depth resolved PMRFI data for Pi/ATP are shown in fig 4(a), 4(b), and 4(c). Figure 4(a) shows a wide variation in superficial metabolite ratios from all infants. Spectra from middle and deep regions, shown in fig 4(b) and (c), show a significant difference between the adverse outcome group and normal infants or asphyxiated infants with a normal outcome. Depth resolved data did not, however, show any increased discrimination compared to global data. The best PMRFI discriminator was the $\mathrm{PCr} / \mathrm{Pi}$ ratio from 2-3 cm below the skull, with sensitivity, specificity, and positive predictive value of $75 \%, 92 \%$, and $75 \%$.

\section{Superficial (a) \\ Pi/ATP}
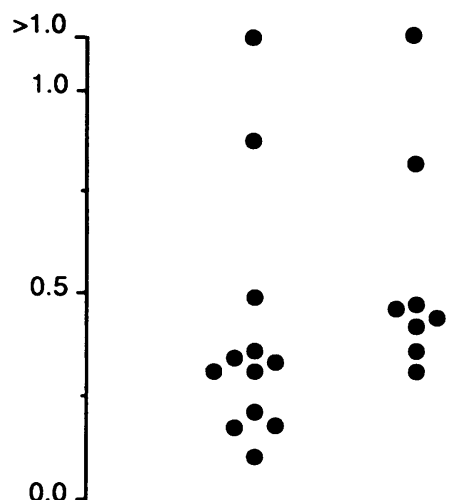

Normal

Asphyxia

(adverse

outcome)

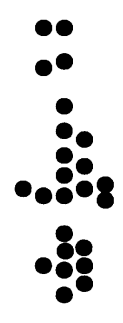

Asphyxia

(normal

outcome)

\section{Discussion}

The majority of infants with clinical and biochemical evidence of birth asphyxia will either show no evidence of neurological abnormality in the newborn period or will exhibit only a mild encephalopathy. ${ }^{7}$ However a minority, about two infants per 1000 livebirths, will develop a moderate or severe hypoxic-ischaemic encephalopathy with seizures and nervous system depression which is variable in degree and duration. ${ }^{8}$ This group of infants has an approximately $50 \%$ incidence of adverse outcome, either death or significant neurodevelopmental delay. $^{7}$

Rational management in the immediate neonatal period, when multiorgan failure requires intensive life support, would be greatly assisted if accurate prognostic tools were available. Simple clinical assessments of encephalopathy grading ${ }^{7}$ and time to attain sucking feeds ${ }^{9}$ are very predictive but retrospective and may be altered by drug treatment. Conventional techniques such as computed tomography ${ }^{10}$ and ultrasound ${ }^{11}$ tend to show definitive changes after the first week of life. Earlier objective evidence of severe irreparable brain damage is important if parents are to be accurately counselled about

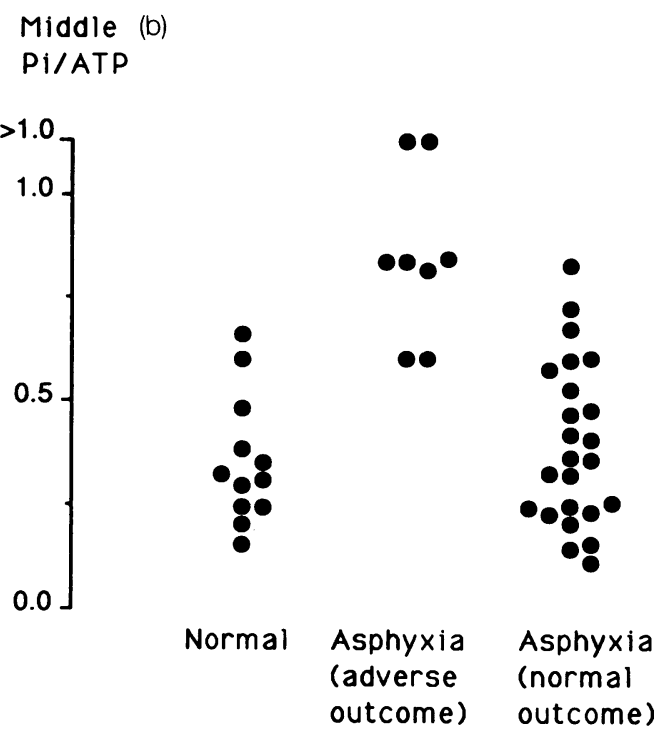

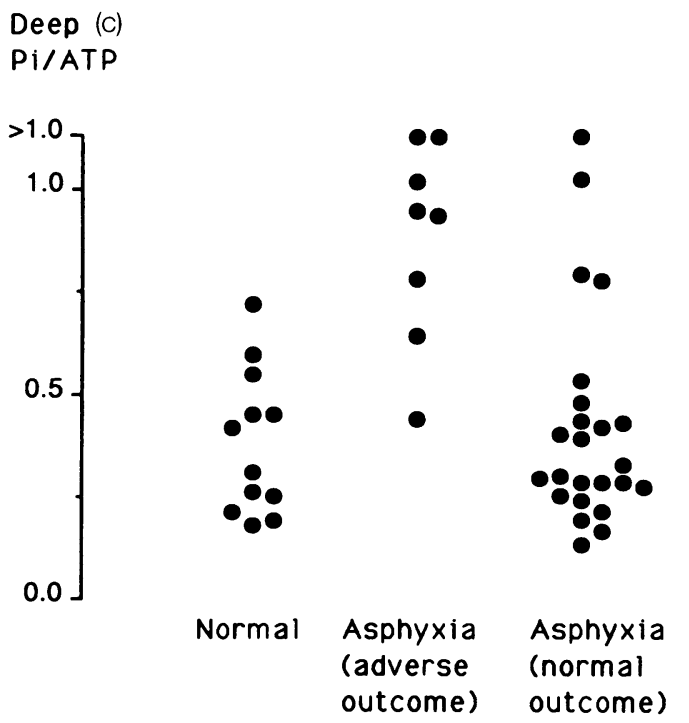

Figure 4 Spatially localised Pi/ATP ratios from superficial (a), middle (b), and deep (c) brain from normal infants and asphyxiated infants divided into groups according to outcome. Infants with adverse outcome are not significantly different from normal infants in superficial regions. They differ from normal infants in middle and deep regions (Mann-Whitney test, $p<0.002$ ) and from asphyxiated infants with a normal outcome $(p<0.001$ and $p<0.002$, middle and deep respectively). 
the wisdom of pursuing intensive care in the first few days of life. Doppler studies of cerebral artery blood flow velocities, ${ }^{12}$ electroencephalography, ${ }^{8}{ }^{31} \mathrm{P} \mathrm{MRS},{ }^{3}$ and more recently near infrared spectroscopy ${ }^{13}$ have been used to assess cerebral status at an early stage, when clinical signs are often heavily masked by sedative and anticonvulsant drugs.

Azzopardi et al have shown that conventional ${ }^{31} \mathrm{P}$ MRS within the first week of life has a high specificity and positive predictive value for the prediction of adverse outcome, so an abnormal magnetic resonance spectrum is clinically helpful. However, a normal $\mathrm{PCr} / \mathrm{Pi}$ ratio is not necessarily reassuring, as reflected in the limited sensitivity reported in their study. ${ }^{3}$ Neonates with apparently normal cerebral energy metabolism in the postasphyxial period may later manifest symptoms suggestive of neuronal damage. The reason may be that conventional surface coil spectroscopy detects magnetic resonance signals from a large and ill defined volume of brain tissue, and the spectrum may be heavily biased by the high signal from superficial tissues. Regions of impaired energy metabolism may therefore escape detection in the global spectrum, especially if they lie deeper in the brain.

PMRFI is one of a variety of techniques for spatial localisation of MRS data, ${ }^{14}$ and its use for the investigation of regional cerebral metabolism in the neonate has been fully described in relation to severity of neonatal encephalopathy. ${ }^{5}$ That study showed that the maximum region of energy impairment in severely asphyxiated full term infants corresponds approximately to subcortical white matter, and suggested that localised spectra from this particularly vulnerable region should be of greater prognostic value than global data. The present study explores that hypothesis by comparing global and regional metabolite ratios as indicators of short term neurodevelopmental outcome for a group of asphyxiated neonates. Only one hemisphere was studied, as none had ultrasound evidence of focal abnormality or asymmetry. There are obvious limitations to this study in respect of the small numbers of subjects, especially controls, and the short duration of follow up. Ninety two percent of asphyxiated infants have received regular clinical examinations in hospital based follow up clinics, but have not been psychometrically or neurodevelopmentally assessed by independent observers, and the youngest are only 15 months old. It is therefore possible that mild or even moderate neurodevelopmental deficits, especially in cognitive areas, may subsequently become apparent in the group designated as having a normal outcome in this study. However, infants in this group are very unlikely to have significant cerebral palsy, in contrast to the adverse outcome group, of whom the two survivors both have severe cognitive and motor deficits.

Figure 3(a) confirms previous studies that show that a low global $\mathrm{PCr} / \mathrm{Pi}$ ratio, implying impaired cerebral energy metabolism, is strongly associated with adverse outcome. In this study, global $\mathrm{Pi} / \mathrm{ATP}$ ratio seemed to discriminate slightly better than $\mathrm{PCr} / \mathrm{Pi}$ between the groups of asphyxiated infants with normal and adverse outcome. This unexpected finding may result from the small numbers of infants in this study. Theoretically, $\mathrm{PCr}$ concentrations would be expected to fall before ATP depletion occurred, so the $\mathrm{PCr} / \mathrm{Pi}$ ratio should be a more sensitive index of abnormality than $\mathrm{Pi} / \mathrm{ATP}$ ratio. In this study, only one severely asphyxiated infant showed significant ATP depletion. This infant was studied at 3 days and died at 6 days and the magnetic resonance spectra was grossly abnormal with no detectable PCr or ATP. As reported previously in a smaller group of infants, spatially localised data clearly show a wide variation in metabolite ratios from superficial brain in both normal and asphyxiated infants. ${ }^{5}$ This is unlikely to be artefactual because the superficial PMRFI spectra have the best signal to noise ratio. Energy impairment was most apparent in deeper brain tissue in the group with subsequent adverse outcome. The sample volumes interrogated to provide the depth resolved spectra do not conform to anatomical boundaries, so it is impossible to be precise about which tissues are represented in each spectrum. Superficial spectra will contain signals from cortex as well as subcortical white matter, middle spectra will represent predominantly subcortical white matter, and deep spectra will also contain signals from periventricular areas.

Prognostic sensitivity of the global metabolite ratios was high and was not improved by obtaining regional information using PMRFI. Only one infant with an adverse outcome had global $\mathrm{PCr} / \mathrm{Pi}$ and $\mathrm{Pi} / \mathrm{ATP}$ ratios in the normal range, perhaps because the MRS study was delayed until 9 days. All the spatially localised metabolite ratios from that infant were also within normal limits. These results confirm that conventional ${ }^{31} \mathrm{P}$ MRS can be a useful prognostic technique after birth asphyxia. In this small study of full term infants, the depth resolved information provided by PMRFI did not improve prediction of adverse outcome.

We are grateful to Dr Andrew Wilkinson, the nursing and medical staff of the neonatal unit, the staff of the magnetic resonance facility, and the parents of the babies studied.

The study would not have been possible without a generous grant from Action Research (who funded Drs Moorcraft and Smyth). The magnetic resonance facility is supported by the Medical Research Council and the Department of Health.

1 Gadian DG. Nuclear magnetic resonance and its application to living systems. Oxford: Clarendon Press, 1980.

2 Hope PL, Costello AMdeL, Cady EB, et al. Cerebral energy metabolism studied with phosphorus NMR spectroscopy in 366-70.

3 Azzopardi D, Wyatt JS, Cady EB, et al. Prognosis of newborn infants with hypoxic-ischemic brain injury assessed by infants with hypoxic-ischemic brain injury assessed by
phosphorus magnetic resonance spectroscopy. Pediatr Res
$1989 ; 25: 445-51$

4 Blackledge MJ, Rajagopalan B, Oberhaensli RD, Bolas NM, metabolism by 31P. Quantitative studies of human cardiac metabolism by $31 \mathrm{P}$ rotati

5 Moorcraft J, Bolas NM, Ives NK, et al. Spatially localised magnetic resonance spectroscopy of the brains of normal magnetic resonance spectroscopy of the brains of no
and asphyxiated newborns. Pediatrics 1991;87:273-82.

6 Cox SJ, Styles P. Towards biochemical imaging. Fournal of Magnetic Resonance 1980;40:209-12.

7 Levene MI, Grindulis H, Sands C, Moore JR. Comparison of two methods of predicting outcome in perinatal asphyxia.
two Lancet 1986; i:67-8.

8 Sarnat HB, Sarnat MS. Neonatal encephalopathy following fetal distress. Arch Neurol 1976;33:696-705.

9 Curtis PD, Matthews TG, Clarke TA, et al. Neonatal seizures: 
the Dublin collaborative study. Arch Dis Child 1988;63: 1065-8.

10 Lipp-Zwahlen AE, Deonna T, Micheli JL, Calame A Chrzanowski R, Cetre E. Prognostic value of neonatal CT scans in asphyxiated term babies: low density score compared with neonatal neurological signs. Neuropediatrics 1985;16:209-17.

11 Babcock DS, Ball W. Postasphyxial encephalopathy in fullterm infants: ultrasound diagnosis. Radiology 1983;148: 417-23.
12 Archer LNJ, Levene MI, Evans DH. Cerebral artery doppler ultrasonography for prediction of outcome after perinatal asphyxia. Lancet 1986;ii:1116-8.

13 Wyatt JS, Edwards AD, Azzopardi D, Reynolds EOR. Magnetic resonance and near infrared spectroscopy for investigation of perinatal hypoxic-ischaemic brain injury. Arch Dis Child 1989;64:953-63.

14 Aue WP. Localisation methods for in vivo nuclear magnetic resonance spectroscopy. Reviews in Magnetic Resonance in resonance spectroscopy
Medicine

Aspirin to prevent fetal growth retardation

The underlying placental dysfunction is apparently similar in preeclampsia and in idiopathic fetal growth retardation, with placental vascular disease and abnormalities of prostaglandin and thromboxane production. Low dose aspirin could help by its effect on intravascular thrombosis and on thromboxane concentrations, low concentrations of thromboxane being associated with decreased pressor response to angiotension II.

A recent French multicentre trial (S Uzan and colleagues, Lancet 1991;337:1427-31) has shown that low dose aspirin reduces the incidence of fetal growth retardation and of maternal proteinuria.

Patients were enrolled into the trial at 15 to 18 weeks of pregnancy. All had had either fetal growth retardation in their one previous pregnancy or a poor outcome in two previous pregnancies, at least one of which was fetal growth retardation, poor outcome being defined as a small for dates baby, fetal death, or placental abruption. When those given placebo were compared with those treated with either aspirin alone or aspirin plus dipyridamole, the mean birth weight was significantly lower in the placebo group $(2526 \mathrm{~g} v 2751 \mathrm{~g}, \mathrm{p}=0.029,95 \%$ confidence interval for difference 129 to $321 \mathrm{~g}$ ). The mean duration of pregnancy was 36 weeks six days in the placebo group and 37 weeks five days in the treated group $(p=0.05)$. Fetal growth retardation occurred in $26 \%$ of patients given placebo and $13 \%$ of those given treatment. Fetal death and placental abruption were also less common in the treatment group but the numbers were small and the differences did not reach statistical significance. There was, however, a significant reduction in maternal proteinuria $(11 \% v 3 \% \mathrm{p}<0.02)$. The greatest effect of treatment was seen in those mothers who had had a poor outcome in more than one previous pregnancy. A separate trial of aspirin alone against aspirin plus dipyridamole showed no significant differences.

Although the results seem encouraging, the authors are very cautious. They clearly fear misuse of their findings and their conclusion is worth quoting: 'thus, it now seems justifiable to propose aspirin treatment for any patient considered at high risk, even if in her first pregnancy. On the other hand, massive use of aspirin by millions of pregnant women yearly certainly cannot be recommended'. 